A $\mathrm{C}$ G G Bications

Bioorganic \&

Medicinal

Chemistry

Reports

\title{
Synthesis and biological evaluation of some 4-amino-3-mercapto- 1,2,4-triazoles bearing Mannich base
}

\section{Yasemin Dundar ${ }^{1}$, Fatma Kaynak-Onurdag $\oplus^{2}$ and Tijen Onklol $\odot{ }^{1 *}$}

\author{
${ }^{1}$ Department of Pharmaceutical Chemistry, Faculty of Pharmacy, Gazi University, 06330, Ankara, \\ Türkiye \\ ${ }^{2}$ Department of Microbiology Faculty of Pharmacy Trakya University, 22030, Edirne, Türkiye
}

(Received October 26, 2019; Revised November 26, 2019 ; Accepted November 30, 2019)

\begin{abstract}
Bacterial and fungal resistance to almost all antimicrobial agents have been observed in disease causing bacteria and fungi. A series of 3-(\{4-[substitutedbenzylideneamino]-1-(morpholin-4-ylmethyl)-5-thioxo-4,5dihydro-1H-1,2,4-triazol-3-yl $\}$ methyl)-2(3H)-benzothiazolone $(\mathbf{6 a - 6 g})$ were prepared by the reaction of morpholine in the presence of formaldehyde using microwave irradiation with the yield ranging from $50 \%$ to $98 \%$. The newly synthesized compounds were characterized on the basis of elemental analysis, IR and ${ }^{1} \mathrm{H}-\mathrm{NMR}$. All the synthesized compounds were tested for their antibacterial activities against Gram + and Gram - bacteria, their clinical isolates and antifungal activities against Candida species in vitro. Many of these compounds exhibited potent antifungal activity with MIC values in the range (MIC: 32-64 $\mu \mathrm{g} / \mathrm{mL}$ ), while compound 6g (MIC: 32 $\mu \mathrm{g} / \mathrm{mL}$ ) had comparable activity with ampicillin and gentamycin (MIC: $4 \mu \mathrm{g} / \mathrm{mL}$ ) against E.faecalis isolate
\end{abstract}

Keywords: 5-Substituted-4-amino-3-mercapto-1,2,4-triazoles; 2(3H)-benzothiazolone; Mannich base; antibacterial; antifungal; MIC (C2019 ACG Publication. All right reserved.

\section{Introduction}

The discovery, development, and clinical use of antibiotics have decreased substantially from bacterial infections during the $20^{\text {th }}$ century. Misuse of antibiotics have causes microorganisms to gain resistance and major problems have begun to occur in the treatment of infections caused by them. ${ }^{1,2}$ Therefore, to avoid the emergence of resistant bacteria and maintain the progress and results are needed new antibacterial agents. The development of resistance mechanism often adversely affected treatment with antimicrobial compounds. Thus, the design and synthesis of novel classes of antimicrobial compounds is urgently needed.

There has been considerable interest in the chemistry of 2(3H)-benzothiazolone and 1,2,4triazole rings. These two rings are linked by methylene bridges. Various 1,2,4-triazole derivatives have been reported to possess antibacterial ${ }^{3,4,5}$, antifungal ${ }^{3,4,5}$, antiproliferative ${ }^{3,6}$ anticancer $^{7}$, antitubercular ${ }^{8}$, anti-inflammatory ${ }^{9}$ properties. $2(3 \mathrm{H})$-Benzothiazolone containing of triazole Schiff and Mannich bases have been examined with a view to explore their potency as better chemotherapeutic agents. ${ }^{10}$

Mannich bases have been also reported as potential biological agents. Mannich reaction is an important tool for synthesis of novel compounds. Mannich bases are physiologically reactive because of the basic function rendering the molecule soluble in aqueous solvents.

We herein report the synthesis of some new triazole compounds and their antimicrobial activity were screened against different bacterial fungal strains.

*Corresponding author: E-Mail: tijen@gazi.edu.tr 


\section{Experimental}

\subsection{Chemistry}

Chemicals and all the solvents, used in this study, were purchased locally from Aldrich, (Germany), Merck (Germany) and Acros (Germany) Chemical. Synthesis of 2(3H)-benzothiazolone (1) ${ }^{12}$, ethyl-(2(3H)-benzothiazolone-3-yl)acetate (2) ${ }^{11}$, (benzothiazol-2(3H)-one-3-yl)acetic acid(3) ${ }^{13}, 3$ [(4-Amino-5-thioxo-1,2,4-triazol-3-yl)methyl]benzothiazol-2(3H)-one (4) $)^{5}$ and 3-[(4-\{[substituted phenylmethylidene] amino -5-thioxo-1,2,4-triazol-3-yl)methyl]-2(3H)-benzothiazolone $(\mathbf{5 a - 5 g})^{5}$ were accomplished according to the previously reported procedures.

Melting points of the compounds were recorded on an Electrothermal-9200 digital melting points apparatus and are uncorrected. Microwave reaction was carried out in MicroSYNTH Microwave Labstation at $1600 \mathrm{~W}$ ( 2 magnetrons $800 \mathrm{~W}$ x2). (Milestone S.r.l. Italy). The ${ }^{1} \mathrm{H}-\mathrm{NMR}$ spectra was recorded in DMSO- $\mathrm{d}_{6}$ on Bruker $400 \mathrm{MHz}$ NMR spectrometer. Chemical shifts are reported in parts per million relative to internal standard tetramethylsilane. FTIR spectra were measured with a Perkin-Elmer 400 (USA) ATR attachment (32 scans, wavenumber 4000-650 $\mathrm{cm}^{-1}$ ) and analyzed using the Spectrum v2.0 software. Elemental analysis was performed on a Leco 932 CHNS instrument (St. Joseph, MI, USA) and the results were within $\pm 0.4 \%$ of the theoretical values.

\subsubsection{General Synthesis of Compounds $\mathbf{6 a - 6 g}$}

3-[(4-\{[substitutedphenylmethylidene]amino\}-5-thioxo-1,2,4-triazol-3-yl)methyl]-2(3H)-benzothiazolone (5a-5g) $0.01 \mathrm{~mol}$ was dissolved in a mixture of 2-propanol. Then formaldehyde $(37 \%, 0.5 \mathrm{~mL})$ and morpholine $(0.0012 \mathrm{~mol})$ were added to this solution. The reaction mixture was heated in a stirred microwave oven for 10 or $15 \mathrm{~min}$ at $73^{\circ} \mathrm{C}(350 \mathrm{~W})$. After completion of the reaction (monitored by TLC using toluene:methanol, 9:1), and kept overnight at room temperature. The resulting solid was collected by filtration, washed with cold 2-propanol and crystallized in appropriate solvents.

Compound $\boldsymbol{6 a}$ : Yield:68\%. mp: $177-178^{\circ} \mathrm{C}$. Recrystallized from 2-propanol. FTIR-ATR $v$ max $\left(\mathrm{cm}^{-1}\right)$ : $1667(\mathrm{C}=\mathrm{O}) .{ }^{1} \mathrm{H}-\mathrm{NMR}\left(\delta, \mathrm{ppm}, 400 \mathrm{MHz}, \mathrm{DMSO}-\mathrm{d}_{6},\right): 9.85(1 \mathrm{H}, \mathrm{s},=\mathrm{CH}), 7.86(2 \mathrm{H}, \mathrm{d}$, phenyl-H), 7.68 $(1 \mathrm{H}, \mathrm{d}, \mathrm{H} 7), 7.63(1 \mathrm{H}, \mathrm{t}$, phenyl-H), $7.54(2 \mathrm{H}, \mathrm{t}$, phenyl-H), $7.40(1 \mathrm{H}, \mathrm{d}, \mathrm{H} 4), 7.33(1 \mathrm{H}, \mathrm{t}, \mathrm{H} 6), 7.21$ $(1 \mathrm{H}, \mathrm{t}, \mathrm{H} 5), 5.45\left(2 \mathrm{H}, \mathrm{s}, \mathrm{CH}_{2}\right), 5.12\left(2 \mathrm{H}, \mathrm{s}, \mathrm{CH}_{2}\right), 3.52(4 \mathrm{H}, \mathrm{t}$, morpholin- $\mathrm{H}), 2.61(4 \mathrm{H}, \mathrm{t}$, morpholin-H). Anal. calc. for $\mathrm{C}_{22} \mathrm{H}_{22} \mathrm{~N}_{6} \mathrm{O}_{2} \mathrm{~S}_{2}$ : C (56.63\%), $\mathrm{H}$ (4.75\%), N (18.01). Found: C (56.59\%), H (4.94\%), N (17.87).

Compound $\boldsymbol{6} \boldsymbol{b}$ : Yield:50\%. mp: $178-179^{\circ} \mathrm{C}$. Recrystallized from 2-propanol. FTIR-ATR $v$ max $\left(\mathrm{cm}^{-1}\right)$ : $1681(\mathrm{C}=\mathrm{O}) .{ }^{1} \mathrm{H}-\mathrm{NMR}\left(\delta, \mathrm{ppm}, 400 \mathrm{MHz}, \mathrm{DMSO}_{6} \mathrm{~d}_{6}\right)$ : $9.82(1 \mathrm{H}, \mathrm{s},=\mathrm{CH}), 7.94(2 \mathrm{H}, \mathrm{m}$, phenyl-H), 7.67 $(1 \mathrm{H}, \mathrm{d}, \mathrm{H} 7), 7.41-7.30\left(4 \mathrm{H}, \mathrm{m}\right.$, phenyl-H, H4, H6), $7.21(1 \mathrm{H}, \mathrm{t}, \mathrm{H} 5), 5.45\left(2 \mathrm{H}, \mathrm{s}, \mathrm{CH}_{2}\right), 5.04(2 \mathrm{H}, \mathrm{s}$, $\left.\mathrm{CH}_{2}\right), 3.51(4 \mathrm{H}, \mathrm{t}$, morpholin- $\mathrm{H}), 2.60(4 \mathrm{H}, \mathrm{t}$, morpholin- $\mathrm{H})$. Anal. calc. for $\mathrm{C}_{22} \mathrm{H}_{21} \mathrm{FN}_{6} \mathrm{O}_{2} \mathrm{~S}_{2}: \mathrm{C}(54.53 \%)$, H (4.37\%), N (17.34). Found: C (54.13), H (4.46\%), N (17.19\%).

Compound $\boldsymbol{6}$ : : Yield:98\%. mp: 200-201 ${ }^{\circ} \mathrm{C}$. Recrystallized from 2-propanol. FTIR-ATR $v$ max $\left(\mathrm{cm}^{-1}\right)$ : $1675(\mathrm{C}=\mathrm{O}) .{ }^{1} \mathrm{H}-\mathrm{NMR}\left(\delta, \mathrm{ppm}, 400 \mathrm{MHz}, \mathrm{DMSO}_{-} \mathrm{d}_{6}\right): 9.91(1 \mathrm{H}, \mathrm{s},=\mathrm{CH}), 7.80(2 \mathrm{H}, \mathrm{d}$, phenyl-H), 7.68 $(1 \mathrm{H}, \mathrm{d}, \mathrm{H} 7), 7.61(2 \mathrm{H}, \mathrm{d}$, phenyl-H), $7.40(1 \mathrm{H}, \mathrm{d}, \mathrm{H} 4), 7.34(1 \mathrm{H}, \mathrm{t}, \mathrm{H} 6), 7.21(1 \mathrm{H}, \mathrm{t}, \mathrm{H} 5), 5.45(2 \mathrm{H}, \mathrm{s}$, $\left.\mathrm{CH}_{2}\right), 5.04\left(2 \mathrm{H}, \mathrm{s}, \mathrm{CH}_{2}\right), 3.51(4 \mathrm{H}, \mathrm{t}$, morpholin- $\mathrm{H}), 2.60(4 \mathrm{H}, \mathrm{t}, \mathrm{morpholin}-\mathrm{H})$. Anal. calc. for $\mathrm{C}_{22} \mathrm{H}_{21} \mathrm{ClN}_{6} \mathrm{O}_{2} \mathrm{~S}_{2}: \mathrm{C}(52.74 \%), \mathrm{H}(4.22 \%), \mathrm{N}$ (6.77\%). Found: C (54.64\%), H (4.27\%), N (16.89\%).

Compound 6d: Yield:94\%. mp: 200-202 ${ }^{\circ} \mathrm{C}$. Recrystallized from 2-propanol. FTIR-ATR $v$ max $\left(\mathrm{cm}^{-1}\right)$ : $1675(\mathrm{C}=\mathrm{O}) .{ }^{1} \mathrm{H}-\mathrm{NMR}\left(\delta, \mathrm{ppm}, 400 \mathrm{MHz}, \mathrm{DMSO}_{-} \mathrm{d}_{6}\right): 9.90(1 \mathrm{H}, \mathrm{s},=\mathrm{CH}), 7.81(2 \mathrm{H}, \mathrm{d}$, phenyl-H), 7.75 $(2 \mathrm{H}, \mathrm{d}$, phenyl-H), $7.67(1 \mathrm{H}, \mathrm{d}, \mathrm{H} 7), 7.40(1 \mathrm{H}, \mathrm{d}, \mathrm{H} 4), 7.33(1 \mathrm{H}, \mathrm{t}, \mathrm{H} 6), 7.21(1 \mathrm{H}, \mathrm{t}, \mathrm{H} 5), 5.45(2 \mathrm{H}, \mathrm{s}$, $\left.\mathrm{CH}_{2}\right), 5.04\left(2 \mathrm{H}, \mathrm{s}, \mathrm{CH}_{2}\right), 3.51(4 \mathrm{H}, \mathrm{t}$, morpholin-H), $2.60(4 \mathrm{H}, \mathrm{t}$, morpholin-H). Anal. calc. for $\mathrm{C}_{22} \mathrm{H}_{21} \mathrm{BrN}_{6} \mathrm{O}_{2} \mathrm{~S}_{2}$ : C (48.44 \%), H (3.88\%), N (15.41\%). Found: C (48.13\%), H (3.77\%), N (15.32\%). 
Compound $6 \boldsymbol{e}$ : Yield:83\%. mp: 204-205 ${ }^{\circ} \mathrm{C}$. Recrystallized from MeOH-DMF. FTIR-ATR $v$ max $\left(\mathrm{cm}^{-}\right.$ $\left.{ }^{1}\right): 1674(\mathrm{C}=\mathrm{O}) .{ }^{1} \mathrm{H}-\mathrm{NMR}\left(\delta, \mathrm{ppm}, 400 \mathrm{MHz}, \mathrm{DMSO}-\mathrm{d}_{6}\right)$ : $9.76(1 \mathrm{H}, \mathrm{s},=\mathrm{CH}), 7.74(2 \mathrm{H}, \mathrm{d}$, phenyl-H), $7.67(1 \mathrm{H}, \mathrm{d}, \mathrm{H} 7), 7.39(1 \mathrm{H}, \mathrm{d}, \mathrm{H} 4), 7.36-7.30\left(3 \mathrm{H}, \mathrm{m}, \mathrm{H} 6\right.$, phenyl-H), $7.21(1 \mathrm{H}, \mathrm{t}, \mathrm{H} 5), 5.43\left(2 \mathrm{H}, \mathrm{s}, \mathrm{CH}_{2}\right)$, $5.04\left(2 \mathrm{H}, \mathrm{s}, \mathrm{CH}_{2}\right), 3.52(4 \mathrm{H}, \mathrm{t}$, morpholin- $\mathrm{H}), 2.61(4 \mathrm{H}, \mathrm{t}$, morpholin- $\mathrm{H}), 2.40\left(3 \mathrm{H}, \mathrm{s}, \mathrm{CH}_{3}\right)$. Anal. calc. for $\mathrm{C}_{23} \mathrm{H}_{24} \mathrm{~N}_{6} \mathrm{O}_{2} \mathrm{~S}_{2}$ : C (57.48\%); H (5.03\%), N (17.49\%). Found: C (57.13\%); H (4.91\%), N (17.40\%).

Compound 6f: Yield:71\%. mp: 205-206 ${ }^{\circ} \mathrm{C}$. Recrystallized from MeOH-DMF. FTIR-ATR $v$ max $\left(\mathrm{cm}^{-1}\right)$ : $1677(\mathrm{C}=\mathrm{O}) .{ }^{1} \mathrm{H}-\mathrm{NMR}\left(\delta, \mathrm{ppm}, 400 \mathrm{MHz}, \mathrm{DMSO}-\mathrm{d}_{6},\right): 9.60(1 \mathrm{H}, \mathrm{s},=\mathrm{CH}), 7.80(2 \mathrm{H}, \mathrm{d}$, phenyl-H), 7.67 $(1 \mathrm{H}, \mathrm{d}, \mathrm{H} 7), 7.38(1 \mathrm{H}, \mathrm{d}, \mathrm{H} 4), 7.34(1 \mathrm{H}, \mathrm{t}, \mathrm{H} 6), 7.23(1 \mathrm{H}, \mathrm{t}, \mathrm{H} 5), 7.08(2 \mathrm{H}, \mathrm{t}$, phenyl-H), $5.42(2 \mathrm{H}, \mathrm{s}$, $\left.\mathrm{CH}_{2}\right), 5.04\left(2 \mathrm{H}, \mathrm{s}, \mathrm{CH}_{2}\right), 3.86\left(3 \mathrm{H}, \mathrm{s}, \mathrm{OCH}_{3}\right), 3.52(4 \mathrm{H}, \mathrm{t}$, morpholin- $\mathrm{H}), 2.61(4 \mathrm{H}, \mathrm{t}$, morpholin-H). Anal. calc. for $\mathrm{C}_{23} \mathrm{H}_{24} \mathrm{~N}_{6} \mathrm{O}_{3} \mathrm{~S}_{2}$ : C (55.63\%), $\mathrm{H}(4.87 \%), \mathrm{N}$ (16.92\%). Found: $\mathrm{C}(55.42 \%), \mathrm{H}(4.97 \%), \mathrm{N}$ $(16.80 \%)$.

Compound $6 \mathrm{~g}$ : Yield:68\%. mp: $148-149^{\circ} \mathrm{C}$. Recrystallized from 2-propanol. FTIR-ATR $v$ max $\left(\mathrm{cm}^{-1}\right)$ : $1674(\mathrm{C}=\mathrm{O}) .{ }^{1} \mathrm{H}-\mathrm{NMR}\left(\delta, \mathrm{ppm}, 400 \mathrm{MHz}, \mathrm{DMSO}_{-} \mathrm{d}_{6}\right): 9.75(1 \mathrm{H}, \mathrm{s},=\mathrm{CH}), 7.79(2 \mathrm{H}, \mathrm{d}$, phenyl-H), 7.68 $(1 \mathrm{H}, \mathrm{d}, \mathrm{H} 7), 7.56(2 \mathrm{H}, \mathrm{d}$, phenyl-H), $7.39(1 \mathrm{H}, \mathrm{d}, \mathrm{H} 4), 7.32(1 \mathrm{H}, \mathrm{t}, \mathrm{H} 6), 7.21(1 \mathrm{H}, \mathrm{t}, \mathrm{H} 5), 5.43(2 \mathrm{H}, \mathrm{s}$, $\mathrm{CH} 2), 5.04\left(2 \mathrm{H}, \mathrm{s}, \mathrm{CH}_{2}\right), 3.52(4 \mathrm{H}, \mathrm{t}$, morpholin- $\mathrm{H}), 2.61(4 \mathrm{H}, \mathrm{t}$, morpholin- $\mathrm{H}), 1.33\left(9 \mathrm{H}, \mathrm{s}, \mathrm{CH}_{3}\right)$. Anal. calc. for $\mathrm{C}_{26} \mathrm{H}_{30} \mathrm{~N}_{6} \mathrm{O}_{2} \mathrm{~S}_{2}$ : C (59.74\%), H (5.79\%), N (16.08). Found: C (59.70\%), H (5.84\%), N (16.10).

\subsection{Microdilution Method}

Standard strains of P. aeruginosa ATCC 27853, E. coli ATCC 25922, S. aureus ATCC 29213, E. faecalis ATCC 29212, clinical isolates of these microorganisms and C. albicans ATCC 10231, C.krusei ATCC 6258 were included in the study. Resistance in clinical isolates was determined by disc diffusion method according to the guidelines of clinical and laboratory standards institute (CLSI). ${ }^{14}$ Standard powders of ampicillin trihydrate, gentamicin sulfate, amphotericin B and fluconazole were obtained from the manufacturers. Stock solutions were dissolved in $\mathrm{pH} 8$ phosphate buffered saline (PBS) (ampicillin trihydrate) and distilled water (gentamicin sulfate and amphotericin B). All bacterial isolates were subcultured in Mueller Hinton Agar (MHA; Merck) plates and incubated overnight at 37 ${ }^{\circ} \mathrm{C}$. The solutions of the newly synthesized compounds and standard drugs were prepared and diluted at $2048,1024,512, \ldots 0.0625 \mu \mathrm{g} / \mathrm{mL}$ concentrations in the wells of microplates within the liquid media. Bacterial susceptibility testing was performed according to the guidelines of CLSI M100-S18. ${ }^{15}$ The bacterial suspensions used for inoculation were prepared at $10^{5} \mathrm{CFU} / \mathrm{mL}$ by diluting fresh cultures at MacFarland 0.5 density $\left(10^{7} \mathrm{CFU} / \mathrm{mL}\right)$. Suspensions of the bacteria at $10^{5} \mathrm{CFU} / \mathrm{mL}$ concentrations were inoculated to the two-fold diluted solution of the compounds. There were $10^{4} \mathrm{CFU} / \mathrm{mL}$ bacteria in the wells after inoculations. Mueller Hinton Broth (MHB; Merck) was used for diluting the bacterial suspension and for two-fold dilution of the compound. Dimethylsulphoxide (DMSO), PBS, pure microorganisms and pure media were used as control wells. A $10 \mu \mathrm{L}$ bacteria inoculum was added to each well of the microdilution trays. The trays were incubated at $37^{\circ} \mathrm{C}$ and minimum inhibitory concentration (MIC) endpoints were read after $24 \mathrm{~h}$ of incubation. All organisms were tested in triplicate in each run of the experiments. The lowest concentration of the compound that completely inhibits macroscopic growth was determined and MICs were reported. Candida culture suspensions were prepared through the guideline of CLSI M27-A3. ${ }^{16}$ All organisms were tested in triplicate in each run of the experiments. The lowest concentration of the compound that completely inhibits macroscopic growth was determined and MICs were reported. 


\section{Result and Discussion}

\subsection{Chemistry}

The synthesis of 3-\{[(4-(substitued-2-ylmethylidene)-amino-1-(morpholin-4-ylmethyl)-5thioxo-4,5-dihydro-1H-1,2,4-triazol-3-yl]methyl $\}$-2(3H)-benzothiazolone (6a-g) were accomplished as presented in Scheme 1. 3-(\{4-[((substitued-2-ylmethylidene)amino]-5-thioxo-4,5-dihydro-1H-1,2,4triazol-3-yl\} methyl)-2(3H)-benzothiazolone $(\mathbf{5})^{6}$ when treated with morpholine and formaldehyde in presence of 2-propanol gave Mannich bases (6a-g) under microwave irradiation. Advantages of microwave irradiation such as short reaction time, pure product and easy work up prompted us to synthesize compounds.

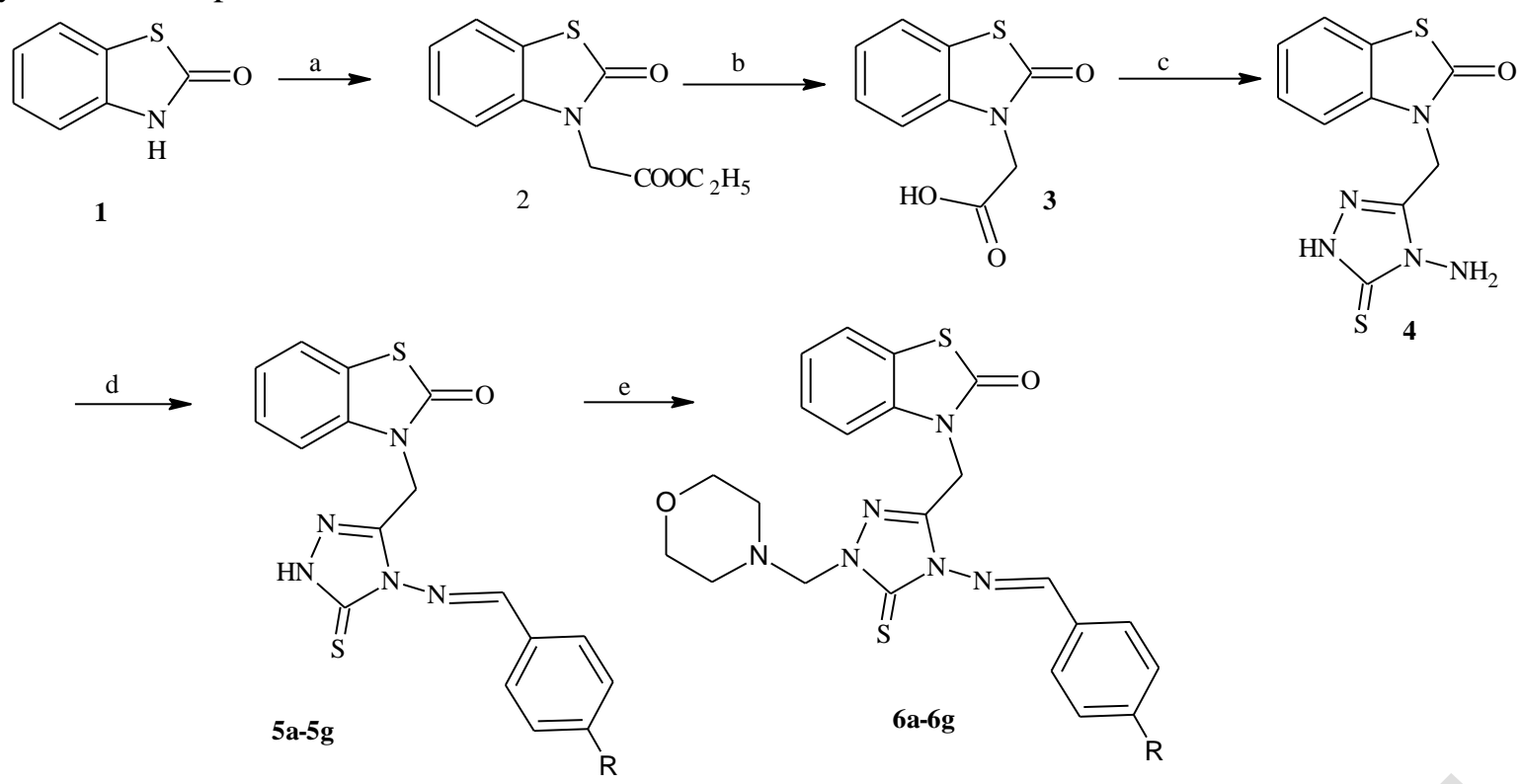

a: $\mathrm{BrCH}_{2} \mathrm{COOC}_{2} \mathrm{H}_{5}, \mathrm{~K}_{2} \mathrm{CO}_{3}$, Aceton, $\mathrm{MW}$; b: $\mathrm{HCl}$, reflux; c: $\mathrm{NH}_{2} \mathrm{NHCSNHNH}_{2}$, fusion;

d: p-substituebenzaldehyde, $\mathrm{CH}_{3} \mathrm{COOH}, \mathrm{MW}$; e: formaldehyde, morpholine, $\mathrm{MW}$

Scheme 1. Synthesis of compounds 6a-6g

The structures of the synthesized compounds were elucidated by IR, ${ }^{1} \mathrm{H}-\mathrm{NMR}$, and elemental analysis. IR spectrum of mannich bases showed an absorption band at $1671-1695 \mathrm{~cm}^{-1}$ characteristic of carbonyl group in the molecule. The ${ }^{1} \mathrm{H}-\mathrm{NMR}$ spectrum of Mannich base $6 \mathbf{6}-6 \mathrm{~g}$ showed the $-\mathrm{NCH}_{2} \mathrm{~N}$ protons resonated as a singlet at 5.04 integrating for two protons. As expected, the ${ }^{1} \mathrm{H}-\mathrm{NMR}$ spectra of compounds 6a-6g displayed a singlet signal belonging to $-\mathrm{CH}_{2}$ group at $5.45-5.42 \mathrm{ppm}$. Moreover, $=\mathrm{CH}$ signal recorded between 9.91 and $9.60 \mathrm{ppm}$ in the ${ }^{1} \mathrm{H}$ NMR spectra of compounds $6 \mathbf{6}-\mathbf{6 g}$.

\subsection{Biological Studies}

\subsubsection{In vitro Antimicrobial Activities}

The antimicrobial properties of the novel compounds were investigated by minimum imhibitory concentration (MIC), with respect to freshly cultured targeted organism. Preliminary screening of new compounds (6a-6g) were tested for their in vitro antibacterial activity against some gram positive bacteria; S. aureus ATCC 29213, methicillin-resistant S. aureus (MRSA, clinical isolate), E. faecalis ATCC 29212, E. faecalis (resistant to vancomycin, clinical isolate), some gram negative bacteria; $E$. coli ATCC 25922, E. coli ATCC 35218, E. coli isolate, which has an extended spectrum beta lactamase enzyme (ES $\beta$ L), P. aeruginosa ATCC 27853, P. aeruginosa (resistant to gentamicin, clinical isolate) and yeast-like fungi; C. albicans ATCC 10231 and C.krusei ATCC 6258 by using broth microdilution method. Ampicillin and gentamicin were used as standard antimicrobial agents and, amphotericin B and 
fluconazole were used as standard antifungal agents (Table 1). Antibacterial activity results of the compounds (5a-5g) was published by Dundar at all. in $2013^{5}$.

As shown in Table 1, the synthesized compounds $(\mathbf{6 a - 6 g})$ exhibited a broad spectrum of activity with MIC values $64-128 \mu \mathrm{g} / \mathrm{mL}$ against both gram-positive and gram-negative bacteria. In general, the synthesized compounds are more active against bacterial isolates than bacteria. The compounds are also more active against Gram-positive bacteria than Gram-negative bacteria, specially, against $E$. faecalis .

Table. 1. Minimum inhibitory concentration of the Synthesized Compounds (MICs, $\mu \mathrm{g} / \mathrm{mL}$ )

\begin{tabular}{|c|c|c|c|c|c|c|c|c|c|c|}
\hline Compound & 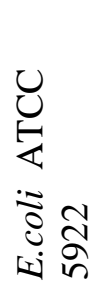 & 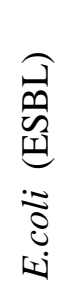 & 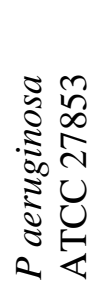 & 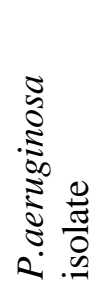 & 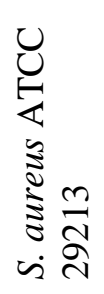 & 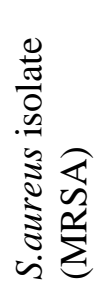 & 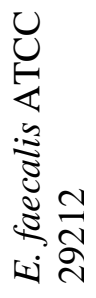 & 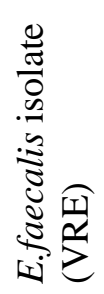 & 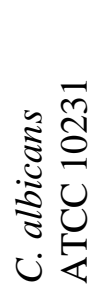 & 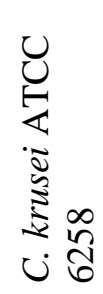 \\
\hline $6 a$ & 64 & 64 & 64 & 64 & 128 & 128 & 64 & 64 & 32 & 32 \\
\hline $6 b$ & 64 & 64 & 64 & 64 & 128 & 128 & 64 & 64 & 32 & 32 \\
\hline $6 c$ & 64 & 64 & 64 & 64 & 64 & 64 & 64 & 64 & 32 & 32 \\
\hline 6d & 64 & 64 & 64 & 64 & 64 & 64 & 64 & 64 & 32 & 32 \\
\hline $6 e$ & 64 & 64 & 64 & 64 & 128 & 128 & 64 & 64 & 64 & 64 \\
\hline $6 f$ & 64 & 64 & 64 & 64 & 128 & 128 & 64 & 64 & 64 & 64 \\
\hline $6 \mathrm{~g}$ & 64 & 64 & 64 & 64 & 64 & 64 & 64 & 32 & 64 & 32 \\
\hline Ampicillin & 8 & 64 & - & - & $<2$ & 32 & $<2$ & 4 & - & - \\
\hline Gentamycin & $<2$ & 64 & $<2$ & 32 & $<2$ & 64 & 4 & 4 & - & - \\
\hline Fluconazole & - & - & - & - & - & - & - & - & 1 & 64 \\
\hline Amphotericin B & - & - & - & - & - & - & - & - & $<0.25$ & 0.5 \\
\hline
\end{tabular}

Regarding the antifungal activity of the synthesized compounds, all compounds showed antifungal activity at 32-64 $\mu \mathrm{g} / \mathrm{mL}$ MIC against both $C$. albicans and $C$. krusei. Compounds $\mathbf{6 a}, \mathbf{6 b}$, $\mathbf{6 c}, \mathbf{6 d}$ and $\mathbf{6 g}$ show inhibitory concentration value of $32 \mu \mathrm{g} / \mathrm{mL}$ against $C$. albicans and $C$. krusei. These compounds have found as effective as the reference drug as Fluconazole.

The Mannich bases (6a-6g) were found to be more active, when compared to Schiff bases (5a5g) derived ${ }^{5}$. The hydrophilic properties of a drug can be further enhanced by its solubility in water, quaternization of the nitrogen atom in the aminomethyl derivative and conversion to an ammonium salt. ${ }^{11}$ This information was found appropriate with our results.

\section{Conclusion}

We have synthesized series of 3-[(4-Amino-5-thioxo-1,2,4-triazol-3-yl)methyl]benzothiazol$2(3 H)$-one bearing Mannich bases. All compounds were evaluated for their antimicrobial activities. The Mannich bases (6a-6g) were shown potent antimicrobial activity compare to Schiff bases derived. Among the synthesized compounds, Mannich bases with phenyl, p-halophenyl and p-ter-butylphenyl substituents showed good antifungal activity as compared to the standard drugs. Hence, it is concluded that, there is sample scope for further study.

\section{Acknowledgements}

This study was supported by Gazi University BAP. Project Number 02/2009-06 and 02/2012- 


\section{ORCID 둥}

Yasemin Dundar: 0000-0002-4378-3629

Fatma Kaynak-Onurdag: 0000-0002-8758-3864

Tijen Onkol: 0000-0003-3973-5728

\section{References}

[1] Chopra, I.; Hodgson, J.; Metcalf, B.; G. Poste, G., The search for antimicrobial agents effective against bacteria resistant to multiple antibiotics. Antimic. Agent. Chemother. 1997, 41 (3), 497-503.

[2] Spellberg, B.; Powers, J.H. ; Brass, E. P.; Miller, L.G. Edwards, J.E. ; Trends in antimicrobial drug development: implications for the future. Antimic. Res. Develop. 2004, 38, 1279-1286.

[3] Popiołek, L.; Rzymowska, J.; Kosikowska, U.; Hordyjewska, A.; Wujec, M.;. Malm, A .; Synthesis, antiproliferative and antimicrobial activity of new Mannich bases bearing 1,2,4-triazole moiety. J. Enz. Inhib. Med. Chem. 2014, 29 (6), 786-795

[4] Popiolek, L.; Biernasiuk, A.; Paruch, K.; Patrejko, P.; Wujec, M.; Synthesis and evaluation of antimicrobial properties of new Mannich bases of 4,5-disubstituted-1,2,4-triazole-3-thiones. Phosphor. Sulfur Sil. Rel. Elem. 2017, 192(7), 880-885.

[5] Dundar, Y.; Onurdag, F.K.; Onkol, T.; Synthesis and biological activity of some 3-[(4-Amino-5-thioxo1,2,4-triazol-3-yl)methyl]benzothiazol-2(3H)-one derivatives. Asian J. Chem. 2013, 25(1), 225-230.

[6] Wang, X.F.; Zhang, S.; Lin, B.L.; Zhao, J.J.; Liu, Y.M.; Zhang, R.L.; Li, B.; Chen, R.L. ; Synthesis and biological evaluation of disulfides bearing 1,2,4-triazole moiety as antiproliferative agents. Med. Chem. Res. 2017, 26 (12), 3367-3374.

[7] Ahmad, A.; Varshney, H.; Rauf, A.; Sherwani, A.; Owais, M.; Synthesis and anticancer activity of long chain substituted 1,3,4-oxadiazol-2-thione, 1,2,4-triazol-3-thione and 1,2,4-triazolo[3,4-b]-1,3,4-thiadiazine derivatives. Arab. J. Chem. 2017, 10 (2), 3347-3357.

[8] Shiradkar, M. R.; Murahari, K. K.; Gangadasu, H. R.; Suresh, T.; Kalyan, C. A.; Panchal, D.; Kaur, R.; Burange, P. ; Ghogare, J.; Mokalec, V. ; Raut, M. ; Synthesis of new S-derivatives of clubbed triazolyl thiazole as anti-Mycobacterium tuberculosis agents. Bioorg. Med. Chem. 2007, 15, 3997-4008.

[9] Paprocka , R.; Wiese, M.; Eljaszewicz, A.; Helmin-Basa, A.; Gzella, A.; Modzelewska-Banachiewicz, B.; Michalkiewicz, J.; Synthesis and anti-inflammatory activity of new 1,2,4-triazole derivatives. Bioorganic and Medicinal Chemistry Letters 2015, 25(13), 2664-2667.

[10] Ciba, M.; Kaynak, F.; Ozgen, S.; Sahin, M.F.; Onkol, T.; Microwave synthesis and antimicrobial evaluation of Mannich bases of 6-Benzoyl-2(3H)-benzothiazolone. Asian J. Chem. 2012, 22(7), 5685-5693.

[11] Saab, A.N.; Sloan, K.B.; Beall, H.D.; Villaneuva, R.; Effect of aminomethyl (N-Mannich base) derivatization on the ability of S-6-acetyloxymethyl-S-mercaptopurine prodrug to deliver 6-mercaptopurine through hairless mouse skin. J. Pharmaceut. Sci. 1990, 79(12), 1099-1104.

[12] Uzun, L.; M.Sc. Thesis, Institute of Health Sciences, Gazi University, 2007.

[13] Onkol, T. ; Dündar, Y. ; Sirmagul, B.; Erol, K.; Sahin, M.F.; “(2-Oxobenzazoline-3-yl)alkanoic acide derivatives and antinociceptive activity. J. Fac. Pharm. Gazi. 2002, 19, 15-24.

[14] Clinical and Laboratory Standards Institute (CLSI) (formerly NCCLS),Performance Standards for Antimicrobial Disk Susceptibility Tests Approved Standard, M2-A9, Clinical and Laboratory Standards Institute, 940 West Valley Road, Wayne, Pennsylvania, USA, 2006.

[15] Clinical and Laboratory Standards Institute (CLSI) (Formerly NCCLS),Performance Standards for Antimicrobial Susceptibility Testing 18th Informational Supplement. CLSI M100-S18, Clinical and Laboratory Standards Institute, 940 West Valley Road, Wayne, Pennsylvania, USA, 2008.

[16] Clinical and Laboratory Standards Institute (CLSI) (formerly NCCLS):Reference Method for Broth Dilution Antifungal Susceptibility Testing yeast; Approved Standard, M27-A3. Clinical and LaboratoryStandarts Institute, 940 West Valley Road, Wayne, Pennsylvania, USA, 2008.

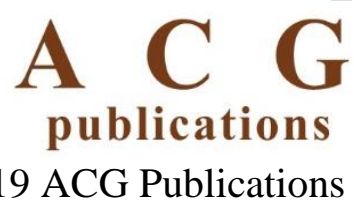

